\title{
Fay's Trisecant Identity and Conformal Field Theory
}

\author{
A. K. Raina \\ Theoretical Physics Group, Tata Institute of Fundamental Research, \\ Homi Bhabha Road, Bombay 400005, India
}

\begin{abstract}
We study the correlation functions of a system of free chiral fermions on a compact Riemann surface using techniques of algebraic geometry. Fay's trisecant identity arises as a consequence of the proof of the uniqueness of correlation functions.
\end{abstract}

\section{Introduction}

The remarkable identity discovered by Fay [1] (see also [2]), and known as the trisecant identity, has been found to play a fundamental role in the theory of Jacobian varieties [3]. In this paper we shall prove Fay's identity by studying a problem in conformal field theory. It has recently been observed by physicists that the trisecant identity arises naturally in the study of conformal field theories on a compact Riemann surface [4]. The trisecant identity was obtained in [4] as a result of computing the four point correlation function of a system of free chiral fermions on a compact Riemann surface by bosonization of the fermions, on the one hand, and by "Wick's theorem," on the other. An alternative approach, using Witten's idea of multiplicative Ward identities [5], was given in a recent paper by S. Sen and the present author [6] (see also [7]).

In the present work we make a detailed analysis of the geometry of the correlation functions of the free field theory of a conjugate pair $\psi, \tilde{\psi}$ of chiral fermions on a compact Riemann surface. The starting point of our analysis is the work of Friedan, Martinec, and Shenker [8] who have provided a local analysis of the correlation functions through operator product expansions. Their analysis provides us with the singularity structure (poles and zeros) of the correlation functions arising from the physics of the problem. Our basic postulate is that the correlation functions have no poles other than those following from this analysis.

We then study the global form of the correlation functions using techniques of algebraic geometry. We find that the line bundle of which a given correlation function is a meromorphic section has a very natural and simple structure. It is the tensor product of the line bundle which is defined by the singularity structure 
demanded by physics (i.e. the analysis of [8]) and a line bundle containing global information. By studying this latter bundle we are able to show that the correlation functions are uniquely determined by our basic postulate.

As an immediate consequence of the uniqueness of the correlation functions we obtain a rigorous proof of Fay's trisecant identity. The physical interpretation of the trisecant identity which emerges from our analysis is that it expresses "Wick's theorem" in this problem. We stress, however, that this does not in any way contradict the remarkable interpretation [4] of the trisecant identity as an expression of the boson-fermion correspondence.

An interesting feature of our analysis is that it is independent of the theory of theta functions. In fact most of our arguments are applicable to an algebraic curve over an arbitrary algebraically closed field. Function theory is brought in only to write down explicitly holomorphic and meromorphic sections of line bundles. We shall not, however, insist upon this aspect in this paper.

Our notations are, for the most part, standard. We refer to [9] for standard facts concerning compact complex manifolds and Riemann surfaces and to [10] for the sheaf cohomology techniques used. All line bundles appearing are holomorphic bundles. We use the same symbol to denote a holomorphic line bundle and its associated sheaf of germs of holomorphic sections. If $D$ is a divisor (e.g. a subvariety of codimension one) in a compact connected complex manifold, we denote the associated holomorphic line bundle as well as its sheaf of germs of holomorphic sections by $\mathcal{O}(D)$. The word unique, when referring to a section, will always mean unique up to multiplication by a nonzero complex number, unless the section is qualified as normalised. Thus $\mathcal{O}(D)$ has a unique meromorphic section with divisor $D$. The set of all holomorphic line bundles forms a group, called the Picard group. The abelian multiplication law is the tensor product $\otimes$, the inverse of an element $\zeta$ (a holomorphic line bundle) is the dual bundle, which we denote by $\zeta^{-1}$, and the identity is the trivial line bundle. Isomorphism of line bundles is denoted by the equality sign. $H^{i}(S, \mathscr{F})$ denotes the $i^{\text {th }}$ cohomology group of $S$ with coefficients in the sheaf $\mathscr{F}$, where $S$ is a compact complex manifold or, more generally, a scheme. Its dimension is denoted by $h^{i}(S, \mathscr{F})$.

We denote by $M$ a given compact connected Riemann surface without boundary of genus $g \geqq 2$ and its $\operatorname{Picard}$ group by $\operatorname{Pic}(M)$. $\operatorname{Pic}^{d}(M)$ denotes the subset of $\operatorname{Pic}(M)$ of holomorphic line bundles of degree $d \in \mathbb{Z}$. The cases $d=0$ and $d=g-1$ are of particular interest. $\operatorname{Pic}^{0}(M)$ is a $g$-dimensional complex torus known as the Picard variety [9]. The multiplication law of $\operatorname{Pic}(M)$ gives an action of $\mathrm{Pic}^{0}(M)$ on $\mathrm{Pic}^{d}(M)$ which is transitive and free. Choosing a fixed element $\zeta \in \operatorname{Pic}^{d}(M)$ we obtain a bijective correspondence between $\operatorname{Pic}^{\circ}(M)$ and $\operatorname{Pic}^{d}(M)$. Thus $\operatorname{Pic}^{d}(M)$ is a $g$-dimensional compact complex manifold. As customary, $K$ denotes the canonical (holomorphic cotangent) bundle of $M$.

\section{The $\psi-\tilde{\psi}$ System for Twisted Fermions}

The system we consider is a two component chiral fermion theory on $M$, whose action is given by [5]

$$
S=\frac{i}{\pi} \int_{M} \tilde{\psi} \bar{D} \psi
$$


Taking local complex coordinates on $M$ in which the metric is of the form $\lambda(z, \bar{z}) d z d \bar{z}$, the operator $\bar{D}$ is essentially $\bar{\partial} \equiv \partial / \partial \bar{z}$.

Since $M$ is a manifold, a precise definition of $\psi$ requires the specification of a spin structure on $M$. Spin structures on $M$ correspond bijectively to the $4^{g}$ isomorphism classes of holomorphic line-bundles $L \in \operatorname{Pic}^{g-1}(M)$ with $L^{2}=K$ [11]. A spin $1 / 2$ fermion field is thus an operator-valued section of one of the $4^{g}$ theta characteristics.

We shall, however, consider the more general case of what is often called a twisted fermion. Thus we shall assume that $\psi$ is an operator valued section of an arbitrary element $\alpha \in \mathrm{Pic}^{g-1}(M)$. Then the invariance of (2.1) requires that $\tilde{\psi}$ be associated with the line bundle $K \otimes \alpha^{-1} \in \mathrm{Pic}^{g-1}(M)$. The only restriction that we shall impose on $\alpha$ is that it should not give rise to zero modes. From (2.1) we see that the mathematical expression of this condition is that $\alpha$ should have no holomorphic sections:

$$
H^{0}(M, \alpha)=0 .
$$

By the Riemann-Roch theorem [9] this means that also

$$
H^{0}\left(M, K \otimes \alpha^{-1}\right)=0 .
$$

Remark 2.1. The condition $(2.2 \mathrm{a}, \mathrm{b})$ will be unnecessarily restrictive in many parts of our analysis. In the following we shall denote an arbitrary element of $\mathrm{Pic}^{g-1}(M)$ by $\xi$, while $\alpha$ will always denote one which satisfies $(2.2 \mathrm{a}, \mathrm{b})$.

Let $Q, P$ denote two points on $M$ which lie in the same coordinate patch with $Q \sim P$. From [8] we have the following operator product expansions (which are to be understood inside some correlation function):

$$
\begin{aligned}
& \psi(Q) \psi(P) \sim O(Q-P), \\
& \tilde{\psi}(Q) \tilde{\psi}(P) \sim O(Q-P), \\
& \tilde{\psi}(Q) \psi(P) \sim \frac{1}{Q-P} .
\end{aligned}
$$

We use (2.3a-c) only in a weak form to determine the zeros and poles of the $2 n$-point function $\left\langle\tilde{\psi}\left(Q_{1}\right) \psi\left(P_{1}\right) \ldots \tilde{\psi}\left(Q_{n}\right) \psi\left(P_{n}\right)\right\rangle$ at coincident points and we postulate that it has no other poles. We make no hypothesis on the residues nor on the existence of other zeros.

\section{Two-Point Function: Local Analysis}

Let $R, S$ be two compact connected complex manifolds and $p_{R}, p_{S}$ the canonical projections from $R \times S$ to $R, S$ respectively. Let $\zeta, \eta$ be holomorphic line bundles over $R, S$ respectively. Then we denote by $\zeta \otimes \eta$ the holomorphic line bundle $p_{R}^{*}(\zeta) \otimes p_{S}^{*}(\eta)$ over $R \times S$ (* denotes the pullback). We take two copies $M_{1}, M_{2}$ of $M$ and denote by $p_{1}, p_{2}$ the canonical projections from $M_{1} \times M_{2}$ defined by $p_{1}(Q, P)=Q, p_{2}(Q, P)=P$. The operator product expansion (2.3) motivates the following definition. 
Definition. Let $\alpha \in \operatorname{Pic}^{g-1}(M)$ satisfy (2.2). The two-point function $\langle\tilde{\psi}(Q) \psi(P)\rangle$ is a meromorphic section of $\left(K \otimes \alpha^{-1}\right) \otimes \alpha$ whose polar divisor is the diagonal $\Delta$ of $M_{1} \times M_{2}$.

We study the line bundle $\left(K \otimes \xi^{-1}\right) \otimes \xi$ in Sect. 4, prove the uniqueness of the two-point function in Sect. 5, and determine the latter in Sect. 6.

\section{Two-Point Function: Global Analysis}

Let $\mathscr{E}$ denote the subset of $\mathrm{Pic}^{g-1}(M)$ consisting of holomorphic line bundles of degree $g-1$ which have a nonzero holomorphic section. Denoting by $M_{d}$ the $d$-fold symmetric product of $M, \mathscr{E}$ is the image of $M_{g-1}$ under the Abel-Jacobi map, which is birational on its image. Hence $\mathscr{E}$ is a divisor of $\operatorname{Pic}^{g-1}(M)$ and defines a line bundle $\mathcal{O}(\mathscr{E})$ on $\mathrm{Pic}^{g-1}(M)$. It follows from the Riemann-Roch theorem that $\mathscr{E}$ is invariant under the involution

of $\mathrm{Pic}^{g-1}(M)$.

$$
i: \zeta \rightarrow K \otimes \zeta^{-1}
$$

For our analysis of the two-point function we require the following lemma which is essentially contained in [12]:

Lemma 4.1. Let $\eta$ be a line bundle on $M$ of degree $\mathrm{g}-2$. Let $I_{\eta}: M \rightarrow \mathrm{Pic}^{g-1}(M)$ be the map defined by

$$
P \rightarrow \eta \otimes \mathcal{O}(P) \text {. }
$$

Then the pullback to $M$ by $I_{\eta}$ of the line bundle $\mathcal{O}(\mathscr{E})$ is $K \otimes \eta^{-1}$.

Proof. We sketch the proof. Since $\eta \in \operatorname{Pic}^{g-2}(M), K \otimes \eta^{-1} \in \operatorname{Pic}^{g}(M)$ so that by Riemann-Roch $K \otimes \eta^{-1}$ has at least one holomorphic section. Assume that $K \otimes \eta^{-1}$ is nonspecial, i.e. $h^{0}\left(M, K \otimes \eta^{-1}\right)=1$. Then $K \otimes \eta^{-1}$ is the image in $\mathrm{Pic}^{g}(M)$ of the unique element $P_{1}+\ldots+P_{g}$ of $M_{g}$ under the birational Abel-Jacobi map. Now

$$
\begin{aligned}
I_{\eta}^{-1}(\mathscr{E}) & =\left\{P \in M \mid h^{0}(M, \eta \otimes \mathcal{O}(P)) \neq 0\right\} \\
& =\left\{P \in M \mid h^{0}\left(M, K \otimes \eta^{-1} \otimes \mathcal{O}(-P)\right) \neq 0\right\} \\
& =\left\{P_{1}, \ldots, P_{g}\right\} .
\end{aligned}
$$

Hence $I_{\eta}^{*}(\mathcal{O}(\mathscr{E}))=\mathcal{O}\left(P_{1}+\ldots+P_{g}\right)=K \otimes \eta^{-1}$.

We can complete the proof by noting that nonspecial elements form a dense open set in $\operatorname{Pic}^{g}(M)$ by the birationality of the Abel-Jacobi map $M_{g} \rightarrow \operatorname{Pic}^{g}(M)$.

Theorem 4.2. Let $\xi \in \operatorname{Pic}^{g-1}(M)$. Consider the map $\phi_{\xi}: M_{1} \times M_{2} \rightarrow \operatorname{Pic}^{g-1}(M)$ given by the composition

$$
(Q, P) \rightarrow \mathcal{O}(Q-P) \in \operatorname{Pic}^{0}(M) \rightarrow \mathcal{O}(Q-P) \otimes \xi \in \operatorname{Pic}^{g-1}(M) .
$$

Let

$$
L_{\xi} \equiv \phi_{\xi}^{*}(\mathcal{O}(\mathscr{E}))
$$

Then

$$
L_{\xi}=\left(K \otimes \xi^{-1}\right) \otimes \xi \otimes \mathcal{O}(\Delta)
$$

as line bundles on $M_{1} \times M_{2}$. 
Remark 4.3. For the proof we use the well-known seesaw theorem in the following form: if $X$ and $Y$ are compact connected complex manifolds and $L$ is a holomorphic line bundle on $X \times Y$ such that for each $x \in X$ the restriction $L \mid\{x\} \times Y$ is trivial and for each $y \in Y$ the restriction $L \mid X \times\{y\}$ is trivial, then $L$ is trivial. This form follows from the statement given in [13].

Proof of Theorem 4.2. We first compute the restriction of the two sides of (4.4) to $\{Q\} \times M_{2}$. The restriction of $\mathcal{O}(\Delta)$ is $\mathcal{O}(Q)$, that of $p_{1}^{*}\left(K \otimes \xi^{-1}\right)$ is trivial (restriction to a fibre), while that of $p_{2}^{*}(\xi)$ is $\xi$. Hence the restriction of the right-hand side of (4.4) is the line bundle $\xi \otimes \mathcal{O}(Q)$ on $M_{2}$.

The restriction of $L_{\xi}$ to $\{Q\} \times M_{2}$ is the pullback of $\mathscr{O}(\mathscr{E})$ by the map $M_{2} \rightarrow \operatorname{Pic}^{g-1}(M)$ given by $P \rightarrow \mathcal{O}(Q-P) \otimes \xi$. Since $\mathscr{E}$ is invariant under the involution $i$ of (4.1), we can take the pullback after involution by $i$, i.e. by the map

$$
P \rightarrow K \otimes \mathcal{O}(P-Q) \otimes \xi^{-1} \text {. }
$$

This map is of the form $P \rightarrow \mathcal{O}(P) \otimes \eta$ with $\eta=K \otimes \xi^{-1} \otimes \mathcal{O}(-Q) \in \operatorname{Pic}^{g-2}(M)$. Applying Lemma 4.1 we conclude that the pullback of $\mathcal{O}(\mathscr{E})$ is $K \otimes \eta^{-1}=\xi \otimes \mathcal{O}(Q)$. Hence (4.4) holds for the restriction to $\{Q\} \times M_{2}$. A similar argument (the involution is not now necessary) shows that (4.4) holds when both sides are restricted to $M_{1} \times\{P\}$. The proof is now complete from Remark 4.3.

We shall later find it convenient to have Theorem 4.2 in the following form:

Corollary 4.4. Let $R$ be a compact connected complex manifold and let $\pi_{1}, \pi_{2}, \pi_{12}$ be canonical projections from $R \times M_{1} \times M_{2}$ defined by $\pi_{1}(r, Q, P)=Q$, $\pi_{2}(r, Q, P)=P, \pi_{12}(r, Q, P)=(Q, P)$. Let $\xi, L_{\xi}, \Delta$ be as in Theorem 4.2. Then,

$$
\pi_{12}^{*}\left(L_{\xi}\right)=\pi_{1}^{*}\left(K \otimes \xi^{-1}\right) \otimes \pi_{2}^{*}(\xi) \otimes \pi_{12}^{*}(\mathcal{O}(\Delta))
$$

as line bundles on $R \times M_{1} \times M_{2}$.

\section{Two-Point Function: Uniqueness}

If $D$ is a divisor of a compact connected complex manifold, then the line bundle $\mathcal{O}(D)$ has a unique meromorphic section whose divisor is $D$ [9]. We then see from Theorem 4.2 that the two point function is unique if $h^{0}\left(M_{1} \times M_{2}, L_{\xi}\right)=1$. We shall now show that this holds when $\xi=\alpha$, where $\alpha$ satisfies the condition (2.2) of no zero modes.

The following lemma is one which we shall use repeatedly. It is elementary and well known to geometers, but we give the proof as we have not been able to locate a convenient reference.

Lemma 5.1. Let $M_{1}, M_{2}$ be two copies of $M$. The diagonal $\Delta$ is isomorphic to $M$. Then the restriction of $\mathcal{O}(\Delta)$ to $\Delta$, which we denote $\mathcal{O}(\Delta) \mid \Delta$, is isomorphic to $T_{\Delta}=K_{\Delta}^{-1}$, where $T_{\Delta}$ and $K_{\Delta}$ are respectively the tangent and cotangent bundles of $\Delta$.

Proof. Let $\mathcal{O}$ denote the structure sheaf of $M_{1} \times M_{2}$. Then the restriction $\mathcal{O} \mid \Delta$ is the structure sheaf of $\Delta$. If $\mathscr{I}_{\Delta}$ denotes the ideal sheaf of $\Delta$ we have the obvious exact sequence

$$
0 \rightarrow \mathscr{I}_{\Delta} \rightarrow \mathcal{O} \rightarrow \mathcal{O} \mid \Delta \rightarrow 0
$$


Tensoring (5.1) by $\mathscr{I}_{\Delta}$ we find

$$
\mathscr{I}_{\Delta} / \mathscr{I}_{\Delta}^{2}=\mathscr{I}_{\Delta} \underset{\mathcal{O}}{\bigotimes} \mathcal{O} \mid \Delta
$$

The left-hand side of (5.2) (the co-normal sheaf of $\Delta$ in $M_{1} \times M_{2}$ ) is $K_{\Delta}$ (see [14]). Since $\mathscr{I}_{\Delta}=\mathcal{O}(-\Delta)$ (see e.g. [10]), we find that

$$
K_{\Delta}=\mathcal{O}(-\Delta) \otimes \mathcal{O}|\Delta \equiv \mathcal{O}(-\Delta)| \Delta \text {. }
$$

The lemma follows on taking duals.

The following simple variant of Lemma 5.1 will also be useful in calculations:

Corollary 5.2. Keeping the notation of Lemma 5.1, consider now $R \times M_{1} \times M_{2}$, where $R$ is a compact connected complex manifold. Let proj denote the canonical projection $R \times \Delta \rightarrow \Delta$. Then

$$
\mathcal{O}(R \times \Delta) \mid R \times \Delta=\operatorname{proj}^{*}\left(K_{\Delta}^{-1}\right) .
$$

Proposition 5.3. There is a canonical isomorphism between $H^{0}\left(M_{1} \times M_{2}, \mathcal{O}(\Delta)\right)$ and $H^{0}\left(M_{1} \times M_{2}, \mathcal{O}\right)$. Hence $h^{0}\left(M_{1} \times M_{2}, \mathcal{O}(\Delta)\right)=1$.

Proof. Tensoring the exact sequence $(5.1)$ by $\mathscr{I}_{\Delta}^{-1}=\mathcal{O}(\Delta)$, we obtain the exact sequence

$$
0 \rightarrow \mathcal{O} \rightarrow \mathcal{O}(\Delta) \rightarrow T_{\Delta} \rightarrow 0,
$$

where we have made use of Lemma 5.1. Now $H^{0}\left(M_{1} \times M_{2}, T_{\Delta}\right)=0$, since $T_{\Delta}$ is of negative degree $(g \geqq 2)$. The result now follows from the cohomology exact sequence associated to (5.3).

Remark 5.4. The unique holomorphic section of $\mathcal{O}(\Delta)$, normalised to vanish like $(Q-P)$ for $(Q, P)$ near $\Delta$, is called the prime form $E(Q, P)$.

Proposition 5.5. $H^{0}\left(M_{1} \times M_{2}, L_{\alpha}\right)$ is canonically isomorphic to $H^{0}\left(\Delta, L_{\alpha} \mid \Delta\right)$ if $\alpha$ satisfies (2.2).

Proof. Tensoring the exact sequence (5.3) by $\left(K \otimes \alpha^{-1}\right) \otimes \alpha$ we obtain the exact sequence

$$
0 \rightarrow\left(K \otimes \alpha^{-1}\right) \bigotimes \alpha \rightarrow L_{\alpha} \rightarrow L_{\alpha} \mid \Delta \rightarrow 0 .
$$

By the Künneth formula [15] we see that

$$
H^{i}\left(M_{1} \times M_{2},\left(K \otimes \alpha^{-1}\right) \bigotimes \alpha\right)=0 \quad(i=0,1),
$$

since $\alpha$ satisfies (2.2). The proposition now follows from the cohomology exact sequence associated to (5.4).

The following simple lemma will be useful in our discussion of the four point function by extending the applicability of Proposition 5.5.

Lemma 5.6. If $R$ and $S$ are compact connected complex manifolds and $L$ is $a$ holomorphic line bundle on $S$, then

$$
H^{0}\left(R \times S, \operatorname{proj}_{S}^{*}(L)\right)=H^{0}(S, L),
$$


where proj $_{S}$ is the canonical projection $R \times S \rightarrow S$. Thus putting $S=M_{1} \times M_{2}$ and denoting the canonical projection $R \times M_{1} \times M_{2} \rightarrow M_{1} \times M_{2}$ by $\pi_{12}$,

$$
H^{0}\left(R \times M_{1} \times M_{2}, \pi_{12}^{*}\left(L_{\alpha}\right)\right)=H^{0}\left(M_{1} \times M_{2}, L_{\alpha}\right) .
$$

Proof. An immediate consequence of the Künneth formula since $\operatorname{proj}_{S}^{*}(L)=\mathcal{O}_{R} \otimes L$, where $\mathcal{O}_{R}$ is the structure sheaf of $R$.

Lemma 5.7. The restriction of $L_{\alpha}$, defined over $M_{1} \times M_{2}$, to the diagonal $\Delta$ is the trivial bundle on $\Delta$. The associated sheaf is the structure sheaf $\mathcal{O} \mid \Delta$ of $\Delta$.

Proof. $L_{\alpha}=p_{1}^{*}\left(K \otimes \alpha^{-1}\right) \otimes p_{2}^{*}(\alpha) \otimes \mathcal{O}(\Delta)$. Hence $L_{\alpha}\left|\Delta=K_{\Delta} \otimes \mathcal{O}(\Delta)\right| \Delta$ and the result follows from Lemma 5.1.

Proposition 5.5 and Lemma 5.7 show that $H^{0}\left(M_{1} \times M_{2}, L_{\alpha}\right)$ is canonically isomorphic to $H^{0}(\Delta, \mathcal{O} \mid \Delta)=\mathbb{C}$. We summarize these results in

Theorem 5.8. If $\alpha \in \mathrm{Pic}^{g-1}(M)$ satisfies the condition (2.2) of no zero modes, then

$$
h^{0}\left(M_{1} \times M_{2}, L_{\alpha}\right)=1 \text {. }
$$

Remark 5.9. This theorem implies that the two-point function is unique.

\section{The Prime Form and Szegö Kernel}

We now discuss function theory on $M$ and show how the well-known expression for the two-point function can be retrieved from our analysis. The relevant Riemann surface and theta function theory is discussed in exactly the form we require in [16] to which we refer for details.

We choose on $M$ a (symplectic) basis of $g a$-cycles and $g b$-cycles of $H_{1}(M, \mathbb{Z})$ and a canonical basis of $g$ holomorphic one forms $w_{1}, \ldots, w_{g}$ spanning $H^{0}(M, K)$. Then the Riemann period matrix is in canonical form $[9,16]$.

We define the Albanese variety $[9,16]$ :

$$
\operatorname{Alb}(M)=H^{0}(M, K)^{*} / H_{1}(M, \mathbb{Z}) .
$$

Choosing a base point $P_{0} \in M$, the Albanese map $\mu$ is defined by

$$
\mu: M \rightarrow \operatorname{Alb}(M), \quad Q \rightarrow\left(\int_{P_{0}}^{Q} w_{1}, \ldots, \int_{P_{0}}^{Q} w_{g}\right),
$$

and is a well-defined holomorphic map. The Abel theorem essentially says that $\operatorname{Alb}(M)$ and $\operatorname{Pic}^{0}(M)$ can be identified [16]. If $Q_{1}+\ldots+Q_{n}-P_{1}-\ldots-P_{n}$ is an arbitrary divisor of degree zero of $M$, then the correspondence is defined by

$$
\operatorname{Pic}^{0}(M) \ni \mathcal{O}\left(Q_{1}+\ldots+Q_{n}-P_{1}-\ldots-P_{n}\right) \leftrightarrow \int_{P_{1}+\ldots+P_{n}}^{Q_{1}+\ldots+Q_{n}} w \in \operatorname{Alb}(M),
$$

where $\mathbf{w}=\left(w_{1}, \ldots, w_{g}\right)$ and the integral is a sum of $n$ line integrals whose endpoints can be chosen from $P_{1}, \ldots, P_{n}$ and $Q_{1}, \ldots, Q_{n}$ respectively in any order. Note that the integral in (6.2) is independent of the choice of base point $P_{0}$ and is evaluated modulo the period lattice. We call the common Abelian variety, defined by the equivalence (6.2), the Jacobian variety $J(M)$. We shall continue for $J(M)$ our 
practice of writing the group operation multiplicatively that we have followed for $\operatorname{Pic}^{0}(M)$.

The Riemann theta function $\theta(z)$ now defines its (symmetric) divisor of zeros $\Theta$ in $J(M) . \theta(z)$ is the unique holomorphic section of the corresponding line bundle $\mathcal{O}(\Theta)$ on $J(M)$. A theta function with characteristics is a holomorphic section of the line bundle associated with a translate of $\Theta$ by some element of $J(M)$. The symmetry of $\Theta$ can be expressed in the multiplicative notation of $\operatorname{Pic}^{0}(M)$ as

$$
\Theta=\Theta^{-1} \text {. }
$$

Riemann's vanishing theorem states that [16] there exists $\kappa \in \mathrm{Pic}^{g-1}(M)$ such that

$$
\Theta=\kappa^{-1} \otimes \mathscr{E} \text {. }
$$

Because of (6.4) and the fact that $\Theta$ and $\kappa$ depend on the choice of canonical bases, $\mathscr{E}$ is sometimes called the canonical theta divisor. The symmetry of $\mathscr{E}$ under the involution (4.1), combined with the symmetry (6.3) of $\Theta$, implies that $\kappa^{2}=K$, i.e. that $\kappa$ is a theta characteristic.

The isomorphism between $J(M)$ and $\operatorname{Pic}^{g-1}(M)$ defined by $\kappa$ enables us to express a holomorphic section of $L_{\xi}$ in terms of theta functions. Since $\xi^{-1} \otimes \mathscr{E}$ $=\left(\xi^{-1} \otimes \kappa\right) \otimes \Theta$, we see that $L_{\xi}$ is the pullback to $M_{1} \times M_{2}$ of the line bundle associated with the translated theta divisor $\left(\xi^{-1} \otimes \kappa\right) \otimes \Theta$.

Definition. We denote by $\theta[\xi](z)$ the theta function with characteristics $\kappa \otimes \xi^{-1}$, which is a holomorphic section of the line bundle $\mathcal{O}\left(\left(\kappa \otimes \xi^{-1}\right) \otimes \Theta\right)$ on $J(M)$. We shall also adopt the notation $\theta[\xi]\left(Q_{1}+\ldots+Q_{n}-P_{1}-\ldots-P_{n}\right)$ for

$$
\theta[\xi]\left(\int_{P_{1}+\ldots+P_{n}}^{Q_{1}+\ldots+Q_{n}} \mathbf{w}\right) .
$$

We summarize our discussion:

Proposition 6.1. $\theta[\xi](Q-P)$ is a holomorphic section of $L_{\xi}$.

Remark 6.2. It is important to note that the uniqueness of the theta function as a holomorphic section of $\mathcal{O}(\Theta)$ does not imply the uniqueness of the pullback as a holomorphic section of $L_{\xi}$ on $M_{1} \times M_{2}$.

The Riemann singularities theorem [16] states that the multiplicity of a point $\xi$ of $\mathscr{E}$ is given by

$$
\text { mult }_{\xi} \mathscr{E}=h^{0}(M, \xi) .
$$

Thus in particular (or directly from the definition)

$$
\theta[\xi](0)=0 \text { if and only if } \xi \in \mathscr{E},
$$

and if $\beta$ is a nonsingular point on $\mathscr{E}$, then

$$
\begin{gathered}
h^{0}(M, \beta)=1, \\
\theta[\beta](0)=0, \quad d \theta[\beta](0) \neq 0 .
\end{gathered}
$$

We can even choose $\beta$ to be a, necessarily odd, theta characteristic [2]. Choosing an odd theta characteristic $\beta$ satisfying (6.7), (6.8) we denote by $h_{\beta}$ its unique holomorphic section. By the Jacobi inversion theorem the (effective) divisor of 
zeros $P_{1}+\ldots+P_{g-1}$ of $h_{\beta}$ is the inverse image in $M_{g-1}$ of $\beta \in \mathscr{E}$. We shall now determine the zeros of $\theta[\beta](Q-P)$ by adapting the argument given by Mumford [2] to our notation.

Lemma 6.2. Let $\beta$ be an odd theta characteristic with $\beta=\mathcal{O}\left(P_{1}+\ldots+P_{g-1}\right)$ and $h^{0}(M, \beta)=1$. Then $\theta[\beta](Q-P)=0$ if and only if (a) $Q=P$, or (b) $P=$ some $P_{i}$ $(i=1, \ldots, g-1)$, or (c) $Q=$ some $P_{i}(i=1, \ldots, g-1)$.

Proof. From the Riemann vanishing theorem $\theta[\beta](Q-P)=0$ if and only if $\mathcal{O}(Q-P) \otimes \beta \in \mathscr{E}$, i.e., denoting $\mathcal{O}(Q) \otimes \beta$ by $\delta$, if

$$
h^{0}(M, \delta \otimes \mathcal{O}(-P)) \neq 0 .
$$

By the Riemann-Roch theorem, $h^{0}(M, \delta)=1+h^{0}(M, \beta \otimes \mathcal{O}(-Q))$. If $h^{0}(M, \beta \otimes \mathcal{O}(-Q))=0$, then $\delta$ has a unique holomorphic section with zeros at $Q, P_{1}, \ldots, P_{g-1}$. Then, for (6.9) to hold, this section must vanish at $P$ which gives parts (a) and (b) of the lemma. If $h^{0}(M, \beta \otimes \mathcal{O}(-Q)) \neq 0$, then $h_{\beta}$ must vanish at $Q$, which gives part (c) of the lemma.

By the Künneth formula, $h_{\beta}(Q) h_{\beta}(P)$ is a holomorphic section of $\left(K \otimes \beta^{-1}\right) \otimes \beta$. Hence from Lemma 6.2 and Theorem 4.2, $\theta[\beta](Q-P) / h_{\beta}(Q) h_{\beta}(P)$ is a holomorphic section of $\mathcal{O}(\Delta)$. Thus, fixing the normalisation of $h_{\beta}$, we have proved:

Proposition 6.3. Following the notation of Lemma 4.2, the prime form $E(Q, P)$ (defined in Remark 5.4) is given by the expression

$$
E(Q, P)=\frac{\theta[\beta](Q-P)}{h_{\beta}(Q) h_{\beta}(P)} .
$$

Remark 6.4. Equation (6.10) is the definition of the prime form given in $[1,2]$.

From Propositions 6.1 and 6.3 we obtain an expression for the unique twopoint function $\langle\tilde{\psi}(Q) \psi(P)\rangle$ which coincides with the form given in the literature [17]:

Theorem 6.5. Let $\alpha \in \mathrm{Pic}^{g-1}(M)$ satisfy (2.2) so that $\theta[\alpha](0) \neq 0$. Then the normalised two-point function is given by the Szegö kernel

$$
S_{\alpha}(Q, P)=\frac{\theta[\alpha](Q-P)}{\theta[\alpha](0)} \frac{1}{E(Q, P)} .
$$

\section{Four-Point Function: Local Analysis}

Applying the operator product expansions (2.3) to the four-point function $\left\langle\tilde{\psi}\left(Q_{1}\right) \psi\left(P_{1}\right) \tilde{\psi}\left(Q_{2}\right) \psi\left(P_{2}\right)\right\rangle$, we find that it must have zeros for $P_{1}=P_{2}$ or $Q_{1}=Q_{2}$ and a simple pole whenever $Q_{1}=P_{1}, Q_{1}=P_{2}, Q_{2}=P_{1}$, or $Q_{2}=P_{2}$. Following our basic postulate, we require that globally the four-point function should not have any further poles, though it may have extra zeros.

We require a precise description of the zeros and poles determined by the local analysis above. We take four copies of $M$ denoted $M_{i}(i=1, \ldots, 4)$ and we denote the product manifold $M_{1} \times M_{2} \times M_{3} \times M_{4}$ by $M^{4}$. An element of $M^{4}$ will be denoted as either $\left(Q_{1}, P_{1}, Q_{2}, P_{2}\right)$ or as $\left(z_{1}, z_{2}, z_{3}, z_{4}\right)$. 
We define the following canonical projections:

$$
\begin{gathered}
p_{i}: M^{4} \rightarrow M \quad(i=1, \ldots, 4) \\
\left(z_{1}, z_{2}, z_{3}, z_{4}\right) \rightarrow z_{i} \\
p r_{i j}: M^{4} \rightarrow M_{i} \times M_{j} \quad(i \neq j=1, \ldots, 4) \\
\left(z_{1}, z_{2}, z_{3}, z_{4}\right) \rightarrow\left(z_{i}, z_{j}\right) \\
\pi_{i j}^{1}, \pi_{i j}^{2}: M_{i} \times M_{j} \rightarrow M \quad(i \neq j=1, \ldots, 4) \\
\pi_{i j}^{1}\left(z_{i}, z_{j}\right)=z_{i} ; \quad \pi_{i j}^{2}\left(z_{i}, z_{j}\right)=z_{j} .
\end{gathered}
$$

We denote the diagonal of $M_{i} \times M_{j}(i \neq j)$ by $\Delta_{i j}$, the diagonal of $M_{i} \times M_{j} \times M_{k}$ $(i \neq j \neq k)$ by $\Delta_{i j k}$, and the diagonal of $M^{4}$ by $\Delta_{1234}$. It is important to note that each such diagonal is isomorphic to $M$.

The diagonal $\Delta_{i j}$ is a divisor in $M_{i} \times M_{j}$ and has an associated line bundle $\mathcal{O}\left(\Delta_{i j}\right)$. Defining

$$
D_{i j}=p r_{i j}^{-1}\left(\Delta_{i j}\right)=\Delta_{i j} \times M_{k} \times M_{l} \quad(i \neq j \neq k \neq l),
$$

we see that $D_{i j}$ is a divisor in $M^{4}$. Then

$$
\mathcal{O}\left(D_{i j}\right)=p r_{i j}^{*} \mathcal{O}\left(\Delta_{i j}\right)
$$

We further define the zero divisor

$$
D_{z}=D_{13}+D_{24}
$$

and the polar divisor

$$
D_{p}=D_{12}+D_{14}+D_{23}+D_{34}
$$

The total divisor is

$$
D=D_{p}-D_{z} .
$$

Definition. Let $\alpha \in \operatorname{Pic}^{g-1}(M)$ and also satisfy $(2.2 \mathrm{a}, \mathrm{b})$. We define the four-point function $\left\langle\tilde{\psi}\left(Q_{1}\right) \psi\left(P_{1}\right) \tilde{\psi}\left(Q_{2}\right) \psi\left(P_{2}\right)\right\rangle$ to be a meromorphic section of

$$
F_{\alpha} \equiv p_{1}^{*}\left(K \otimes \alpha^{-1}\right) \otimes p_{2}^{*}(\alpha) \otimes p_{3}^{*}\left(K \otimes \alpha^{-1}\right) \otimes p_{4}^{*}(\alpha)
$$

whose polar divisor is $D_{p}$ and whose zero divisor contains $D_{z}$.

\section{Four-Point Function: Global Analysis}

For a fixed $\xi \in \operatorname{Pic}^{g-1}(M)$ consider the map

$$
\psi_{\xi}: M^{4} \rightarrow \operatorname{Pic}^{g-1}(M)
$$

defined by composing

$$
\begin{aligned}
\left(Q_{1}, P_{1}, Q_{2}, P_{2}\right) & \rightarrow \mathcal{O}\left(Q_{1}-P_{1}+Q_{2}-P_{2}\right) \in \operatorname{Pic}^{0}(M) \\
& \rightarrow \xi \otimes \mathcal{O}\left(Q_{1}-P_{1}+Q_{2}-P_{2}\right)
\end{aligned}
$$


We denote by $M_{\xi}$ the pullback of the canonical theta divisor by this map, i.e.

$$
M_{\xi}=\psi_{\xi}^{*}(\mathscr{E}) \text {. }
$$

Theorem 8.1.

$$
M_{\xi}=F_{\xi} \otimes \mathcal{O}(D),
$$

where $D$ and $F_{\xi}$ were defined in (7.3c) and (7.4) respectively.

Proof. We use the seesaw principle for line bundles on $M^{4}$ with $X=M_{1} \times M_{2}$, $Y=M_{3} \times M_{4}$ (see Remark 4.3).

Fix $Q_{2}, \quad P_{2}$ and restrict all line bundles appearing in (8.3) to $M_{1} \times M_{2} \times\left\{\left(Q_{2}, P_{2}\right)\right\}$. Then it is clear that $\mathcal{O}\left(D_{13}\right), \mathcal{O}\left(D_{24}\right), \mathcal{O}\left(D_{12}\right), \mathcal{O}\left(D_{14}\right)$, and $\mathcal{O}\left(D_{23}\right)$ restrict respectively to $\pi_{12}^{1 *}\left(\mathcal{O}\left(Q_{2}\right)\right), \pi_{12}^{2 *}\left(\mathcal{O}\left(P_{2}\right)\right), \quad \mathcal{O}\left(\Delta_{12}\right), \pi_{12}^{1 *}\left(\mathcal{O}\left(P_{2}\right)\right)$, $\pi_{12}^{2 *}\left(\mathcal{O}\left(Q_{2}\right)\right)$. The restriction of $\mathcal{O}\left(D_{34}\right)$, being a restriction to a fibre, is trivial. Let $J$ denote the line bundle $\mathcal{O}\left(Q_{2}-P_{2}\right)$ on $M$. Then

$$
\mathcal{O}(D) \mid M_{1} \times M_{2} \times\left\{\left(Q_{2}, P_{2}\right)\right\}=\pi_{12}^{1 *}\left(J^{-1}\right) \otimes \pi_{12}^{2 *}(J) \otimes \mathcal{O}\left(\Delta_{12}\right) .
$$

The restriction of $F_{\xi}$ is

$$
\pi_{12}^{1 *}\left(K \otimes \xi^{-1}\right) \otimes \pi_{12}^{2 *}(\xi)
$$

Hence the right-hand side of (8.3) reduces to

$$
\pi_{12}^{1 *}\left(K \otimes \xi^{-1} \otimes J^{-1}\right) \otimes \pi_{12}^{2 *}(\xi \otimes J) \otimes \mathcal{O}\left(\Delta_{12}\right) .
$$

The restriction of $M_{\xi}$ is the pullback to $M_{1} \times M_{2}$ of $\mathscr{O}(\mathscr{E})$ by the map

$$
\left(Q_{1}, P_{1}\right) \rightarrow \mathcal{O}\left(Q_{1}-P_{1}\right) \otimes J \otimes \xi .
$$

By Theorem 4.2 (replacing $\xi$ by $\xi \otimes J$ ),

$$
M_{\xi} \mid M_{1} \times M_{2} \times\left\{\left(Q_{2}, P_{2}\right)\right\}=\pi_{12}^{1 *}\left(K \otimes \xi^{-1} \otimes J^{-1}\right)
$$

which coincides with (8.6).

$$
\otimes \pi_{12}^{2 *}(\xi \otimes J) \otimes \mathcal{O}\left(\Delta_{12}\right),
$$

A similar calculation in which we now restrict to $\left\{\left(Q_{1}, P_{1}\right)\right\} \times M_{3} \times M_{4}$ leads to a similar equality. By the seesaw principle, Theorem 8.1 is established.

\section{Four-Point Function: Uniqueness}

We now study the uniqueness of the four-point function when $\xi=\alpha$, where $\alpha$ satisfies condition (2.2) of no zero modes. From Theorem 8.1 we see that the fourpoint function is (if it exists) obtained by tensoring the unique meromorphic section of $\mathcal{O}(-D)$ with divisor $-D$ with a holomorphic section of $M_{\alpha}$. Thus the question of uniqueness is reduced to computing $H^{0}\left(M^{4}, M_{\alpha}\right)=H^{0}\left(M^{4}, F_{\alpha} \otimes \mathcal{O}(D)\right)$. Unlike in the two-point case, $D$ is not an effective divisor and so the cohomology calculation is more difficult. Nevertheless the remarkable structure of the fourpoint function enables us to reduce the problem to the two-point function case on submanifolds of $M^{4}$.

Lemma 9.1. $H^{i}\left(M^{4}, F_{\alpha} \otimes \mathcal{O}\left(-D_{z}\right)\right)=0$ for $i=0,1$.

Proof. By the Künneth formula

$$
H^{i}\left(M^{4}, F_{\alpha}\right)=0 \quad \text { for } \quad i=0,1
$$


as a consequence of (2.2). The ideal sheaf of $D_{z}$ as a subscheme of $M^{4}$ is $\mathcal{O}\left(-D_{z}\right)$ [10]. Denoting by $\mathcal{O}$ and $\mathcal{O} \mid D_{z}$ the structure sheaves of $M^{4}$ and $D_{z}$ respectively, we have the short exact sequence

$$
0 \rightarrow \mathcal{O}\left(-D_{z}\right) \rightarrow \mathcal{O} \rightarrow \mathcal{O} \mid D_{z} \rightarrow 0 .
$$

Tensoring (9.2) with $F_{\alpha}$ we obtain a new short exact sequence

$$
0 \rightarrow F_{\alpha} \otimes \mathcal{O}\left(-D_{z}\right) \rightarrow F_{\alpha} \rightarrow F_{\alpha} \mid D_{z} \rightarrow 0 \text {. }
$$

The cohomology exact sequence associated to (9.3) now gives us

$$
H^{0}\left(M^{4}, F_{\alpha} \otimes \mathcal{O}\left(-D_{z}\right)\right)=0,
$$

because of (9.1), and the canonical isomorphism

$$
H^{1}\left(M^{4}, F_{\alpha} \otimes \mathcal{O}\left(-D_{z}\right)\right)=H^{0}\left(D_{z}, F_{\alpha} \mid D_{z}\right) .
$$

Recall from (7.3a) that $D_{z}=D_{13}+D_{24}$. Computing $F_{\alpha}\left|D_{13}, F_{\alpha}\right| D_{24}$ we find from the Künneth formula that $H^{0}\left(D_{13}, F_{\alpha} \mid D_{13}\right)=0=H^{0}\left(D_{24}, F_{\alpha} \mid D_{24}\right)$. Hence $H^{0}\left(D_{z}, F_{\alpha} \mid D_{z}\right)=0$.

Proposition 9.2. There is a canonical isomorphism between $H^{0}\left(M^{4}, M_{\alpha}\right)$ and $H^{0}\left(D_{p}, M_{\alpha} \mid D_{p}\right)$.

Proof. Tensoring the exact sequence

$$
0 \rightarrow \mathcal{O}\left(-D_{p}\right) \rightarrow \mathcal{O} \rightarrow \mathcal{O} \mid D_{p} \rightarrow 0
$$

by $F_{\alpha} \otimes \mathcal{O}(D)$, we obtain the result from the associated cohomology exact sequence and Lemma 9.1.

We have thus reduced the problem to studying the restriction of $M_{\alpha}$ to the set defined by the effective divisor $D_{p}=D_{12}+D_{14}+D_{23}+D_{34}$. As we shall show, it is sufficient to study the restriction of $M_{\alpha}$ to each of the divisors $D_{12}, D_{14}, D_{23}, D_{34}$ as well as to their mutual intersections.

We are obliged, at this point, to introduce notation for the numerous canonical projections which appear in our calculations. We denote the canonical projections from $\Delta_{i j} \times M_{k} \times M_{l}(i \neq j \neq k \neq l)$ to $\Delta_{i j}, M_{k} \times M_{l}, M_{k}, M_{l}$ by respectively $\operatorname{proj}_{\Delta}$, $\operatorname{proj}_{k l}, \operatorname{proj}_{k}, \operatorname{proj}_{l}$; from $\Delta_{i j} \times M_{k}$ to $\Delta_{i j}, M_{k}$ by $\pi_{\Delta}, \pi_{k}$ respectively; and from $\Delta_{i j} \times \Delta_{k l}$ to $\Delta_{i j}, \Delta_{k l}$ by $\pi_{i j}$ and $\pi_{k l}$ respectively.

We first compute the restriction of $M_{\alpha}$ to the various $D_{i j}$ of which $D_{p}$ is comprised, and then to their various intersections $D_{i j} \cap D_{k l}$.

Lemma 9.3. Let $D_{i j} \in\left\{D_{12}, D_{14}, D_{23}, D_{34}\right\}$. There is exactly one $D_{k l}$ in the same set such that $i \neq j \neq k \neq l$. Then,

$$
M_{\alpha} \mid D_{i j}=\operatorname{proj}_{k l}^{*}\left(L_{\alpha}^{k, l}\right),
$$

where $L_{\alpha}^{k, l}$ is the line bundle $L_{\alpha}$ of (4.4) constructed over $M_{k} \times M_{l}$.

Proof. To compute $M_{\alpha} \mid D_{i j}$, note that

$$
\begin{aligned}
\mathcal{O}(D) \mid D_{i j} & =\mathcal{O}\left(D_{i j}\right)\left|D_{i j} \otimes \mathcal{O}\left(\Delta_{i j} \times \Delta_{k l}\right)\right| D_{i j} \\
& =\operatorname{proj}_{\Delta}^{*}\left(K_{\Delta}^{-1}\right) \otimes \operatorname{proj}_{k l}^{*}\left(\mathcal{O}\left(\Delta_{k l}\right)\right),
\end{aligned}
$$


where $K_{\Delta}$ is the canonical line bundle of $\Delta_{i j}$. We have used Corollary 5.2 in the second line.

$$
F_{\alpha} \mid D_{i j}=\operatorname{proj}_{\Delta}^{*}\left(K_{\Delta}\right) \otimes \operatorname{proj}_{k}^{*}\left(K \otimes \alpha^{-1}\right) \otimes \operatorname{proj}_{l}^{*}(\alpha) .
$$

Combining (9.7) and (9.8) we obtain the result.

Lemma 9.4. $M_{\alpha}\left|D_{i j} \cap D_{k l}=\mathcal{O}\right| \Delta_{i j} \times \Delta_{k l}$ if $i \neq j \neq k \neq l$, where the right-hand side is the structure sheaf of $\Delta_{i j} \times \Delta_{k l}$. The other cases are as follows:

$$
\begin{array}{ll}
M_{\alpha} \mid D_{12} \cap D_{14}=L_{\alpha}^{3,124} ; & M_{\alpha} \mid D_{12} \cap D_{23}=L_{\alpha}^{123,4} ; \\
M_{\alpha} \mid D_{14} \cap D_{34}=L_{\alpha}^{134,2} ; & M_{\alpha} \mid D_{23} \cap D_{34}=L_{\alpha}^{1,234} .
\end{array}
$$

In addition, $M_{\alpha}\left|\Delta_{1234}=\mathcal{O}\right| \Delta_{1234}=$ structure sheaf of $\Delta_{1234}$. In the above, $L_{\alpha}^{i j k, l}\left(L_{\alpha}^{i, j k l}\right)$ is the line bundle of (4.4) constructed over $\Delta_{i j k} \times M_{l}\left(M_{i} \times \Delta_{j k l}\right)$.

Proof. A straightforward computation by the methods already illustrated.

Proposition 9.5. Let $D_{i j}, D_{k l}$ be any two distinct elements of the set $\left\{D_{12}, D_{14}, D_{23}, D_{34}\right\}$. Then the diagram of homomorphisms

$$
\begin{gathered}
H^{0}\left(D_{i j}, M_{\alpha} \mid D_{i j}\right) \stackrel{a}{\rightarrow} H^{0}\left(\Delta_{1234}, M_{\alpha} \mid \Delta_{1234}\right) \\
H^{0}\left(D_{i j} \cap D_{k l}, M_{\alpha} \mid D_{i j} \cap D_{k l}\right)
\end{gathered}
$$

commutes and each arrow is an isomorphism.

Proof. We choose $D_{i j}$ to be $D_{12}$ and $D_{k l}$ to be successively $D_{34}$ and $D_{23}$. Every other case is similar to one of these cases. By Lemma 9.3,

$$
\begin{aligned}
H^{0}\left(D_{12}, M_{\alpha} \mid D_{12}\right) & =H^{0}\left(\Delta_{12} \times M_{3} \times M_{4}, \operatorname{proj}_{34}^{*}\left(L_{\alpha}^{3,4}\right)\right) \\
& =H^{0}\left(M_{3} \times M_{4}, L_{\alpha}^{3,4}\right),
\end{aligned}
$$

by Lemma 5.6. Choosing $D_{k l}$ to be $D_{34}$, we note that by Theorem 4.2 ,

$$
\begin{aligned}
H^{0}\left(M_{3} \times M_{4}, L_{\alpha}^{3,4}\right) & =H^{0}\left(\Delta_{34}, \mathcal{O} \mid \Delta_{34}\right) \\
& =H^{0}\left(\Delta_{12} \times \Delta_{34}, \pi_{34}^{*}\left(\mathcal{O} \mid \Delta_{34}\right)\right) \\
& =H^{0}\left(\Delta_{12} \times \Delta_{34}, \mathcal{O} \mid \Delta_{12} \times \Delta_{34}\right) \\
& =H^{0}\left(D_{12} \cap D_{34}, M_{\alpha} \mid D_{12} \cap D_{34}\right) \quad \text { by Lemma } 9.4 .
\end{aligned}
$$

Choosing now $D_{k l}$ as $D_{23}$, note that there is a natural isomorphism of $\Delta_{123} \times M_{4}$ with $M_{3} \times M_{4}$ so that continuing from (9.9),

$$
\begin{aligned}
H_{0}\left(M_{3} \times M_{4}, L_{\alpha}^{3,4}\right) & =H^{0}\left(\Delta_{123} \times M_{4}, L_{\alpha}^{123,4}\right) \\
& =H^{0}\left(D_{12} \cap D_{23}, M_{\alpha} \mid D_{12} \cap D_{23}\right) \quad \text { by Lemma } 9.4 .
\end{aligned}
$$

In this way the proposition is proved for the arrow $b$. The isomorphism $c$ follows either directly (when the restriction of $M_{\alpha}$ to $D_{i j} \cap D_{k l}$ is trivial) or from Theorem 4.2. As for the arrow $a$, choose the unique $D_{k l}$ for a given $D_{i j}$ such that $i \neq j$ $\neq k \neq l$. Then we have the proper inclusions $\Delta_{1234} \subset \Delta_{i j} \times \Delta_{k l} \equiv \mathrm{D}_{i j} \cap \mathrm{D}_{k l} \subset \mathrm{D}_{i j}$. This proves that $a$ is an isomorphism from our earlier discussion. 
Proposition 9.6. $h^{0}\left(D_{p}, M_{\alpha} \mid D_{p}\right)=1$.

Proof. Since $\Delta_{1234}$ is contained in each of $D_{12}, D_{14}, D_{23}, D_{34}$ whose sum is $D_{p}$, this induces a natural map

$$
H^{0}\left(D_{p}, M_{\alpha} \mid D_{p}\right) \rightarrow H^{0}\left(\Delta_{1234}, M_{\alpha} \mid \Delta_{1234}\right) .
$$

To show that this map is injective, it suffices to show that a holomorphic section $\sigma$ of $M_{\alpha} \mid D_{p}$ vanishes if it vanishes over $\Delta_{1234}$. Given such a $\sigma$, then by the horizontal arrow $a$ of Proposition 9.5, $\sigma \mid D_{i j}$ vanishes for each of the irreducible divisors whose sum is $D_{p}$. Hence $\sigma \equiv 0$. Since $h^{0}\left(\Delta_{1234}, M_{\alpha} \mid \Delta_{1234}\right)=h^{0}\left(\Delta_{1234}, \mathcal{O} \mid \Delta_{1234}\right)=1$, hence $h^{0}\left(D_{p}, M_{\alpha} \mid D_{p}\right) \leqq 1$.

To show that there is a nonzero section of $M_{\alpha} \mid D_{p}$ over $D_{p}$, take a nonzero section $s$ of $M_{\alpha} \mid \Delta_{1234}$ over $\Delta_{1234}$. Let $s_{i j}$ be the section of $M_{\alpha} \mid D_{i j}$ over $D_{i j}$ which goes into $s$ under the isomorphism $a$ of Proposition 9.5. From the commutative diagram of Proposition 9.5, we see that $s_{i j}$ and $s_{k l}$ coincide on $D_{i j} \cap D_{k l}$. Hence the $\left\{s_{i j}\right\}$ can be patched together to give a (non-zero) section $\sigma$ of $M_{\alpha} \mid D_{p}$ over $D_{p}$. Thus $h^{0}\left(D_{p}, M_{\alpha} \mid D_{p}\right)=1$.

Recalling Proposition 9.2, we now summarise the main result of this section in:

Theorem 9.7. If $\alpha$ satisfies the condition (2.2) of no zero modes, then

$$
h^{0}\left(M^{4}, M_{\alpha}\right)=1 \text {. }
$$

This theorem establishes the uniqueness of the four-point function.

\section{Fay's Trisecant Identity}

We follow the conventions of Sect. 6 for theta functions. Generalising Proposition 6.1 we see that a holomorphic section of $M_{\xi}$ over $M^{4}$ is $\theta[\xi]\left(Q_{1}+Q_{2}-P_{1}-P_{2}\right)$. By Theorem 9.7 this holomorphic section is unique when $\xi=\alpha$. The unique meromorphic section of $\mathcal{O}(-D)$ with divisor $-D$ is

$$
\frac{E\left(Q_{1}, Q_{2}\right) E\left(P_{2}, P_{1}\right)}{E\left(Q_{1}, P_{1}\right) E\left(Q_{1}, P_{2}\right) E\left(Q_{2}, P_{1}\right) E\left(Q_{2}, P_{2}\right)} .
$$

We have thus proved:

Theorem 10.1. Let $\alpha \in \mathrm{Pic}^{g-1}(M)$ satisfy (2.2) so that $\theta[\alpha](0) \neq 0$. The unique normalised four-point function is given by

$$
\begin{aligned}
& \left\langle\tilde{\psi}\left(Q_{1}\right) \psi\left(P_{1}\right) \tilde{\psi}\left(Q_{2}\right) \psi\left(P_{2}\right)\right\rangle=\frac{\theta[\alpha]\left(Q_{1}+Q_{2}-P_{1}-P_{2}\right)}{\theta[\alpha](0)} \\
& \times \frac{E\left(Q_{1}, Q_{2}\right) E\left(P_{2}, P_{1}\right)}{E\left(Q_{1}, P_{1}\right) E\left(Q_{1}, P_{2}\right) E\left(Q_{2}, P_{1}\right) E\left(Q_{2}, P_{2}\right)} \cdot \square
\end{aligned}
$$

Since we are dealing with a theory of free fermions, we expect the four-point function to be given by "Wick's theorem" as a determinant of two point functions:

$$
\left\langle\tilde{\psi}\left(Q_{1}\right) \psi\left(P_{1}\right) \tilde{\psi}\left(Q_{2}\right) \psi\left(P_{2}\right)\right\rangle=\operatorname{det}\left|\begin{array}{ll}
S_{\alpha}\left(Q_{1}, P_{1}\right) & S_{\alpha}\left(Q_{1}, P_{2}\right) \\
S_{\alpha}\left(Q_{2}, P_{1}\right) & S_{\alpha}\left(Q_{2}, P_{2}\right)
\end{array}\right| .
$$

It is clear that the right-hand side of (10.2) is a meromorphic section of $F_{\alpha}$ with the correct local singularity structure. 
Theorem 10.2 (Fay's trisecant identity). Let $\alpha \in \operatorname{Pic}^{g-1}(M)$ with $H^{0}(M, \alpha)=0$. Then for $\left(Q_{1}, P_{1}, Q_{2}, P_{2}\right) \in M^{4}$ :

$$
\begin{aligned}
\frac{\theta[\alpha]\left(Q_{1}+Q_{2}-P_{1}-P_{2}\right)}{\theta[\alpha](0)} \frac{E\left(Q_{1}, Q_{2}\right) E\left(P_{2}, P_{1}\right)}{E\left(Q_{1}, P_{1}\right) E\left(Q_{1}, P_{2}\right) E\left(Q_{2}, P_{1}\right) E\left(Q_{2}, P_{2}\right)} \\
=\operatorname{det}\left|\begin{array}{ll}
S_{\alpha}\left(Q_{1}, P_{1}\right) & S_{\alpha}\left(Q_{1}, P_{2}\right) \\
S_{\alpha}\left(Q_{2}, P_{1}\right) & S_{\alpha}\left(Q_{2}, P_{2}\right)
\end{array}\right| .
\end{aligned}
$$

Proof. We can rewrite (10.3) as:

$$
\begin{aligned}
\frac{\theta[\alpha]\left(Q_{1}+Q_{2}-P_{1}-P_{2}\right)}{\theta[\alpha](0)}= & \frac{E\left(Q_{1}, P_{1}\right) E\left(Q_{1}, P_{2}\right) E\left(Q_{2}, P_{1}\right) E\left(Q_{2}, P_{2}\right)}{E\left(Q_{1}, Q_{2}\right) E\left(P_{2}, P_{1}\right)} \\
& \times \operatorname{det}\left|\begin{array}{ll}
S_{\alpha}\left(Q_{1}, P_{1}\right) & S_{\alpha}\left(Q_{1}, P_{2}\right) \\
S_{\alpha}\left(Q_{2}, P_{1}\right) & S_{\alpha}\left(Q_{2}, P_{2}\right)
\end{array}\right| .
\end{aligned}
$$

Expanding the right-hand side of (10.4), using (6.11) for $S_{\alpha}(Q, P)$, we can easily check that the apparent poles all cancel. More precisely, the right-hand side is holomorphic in the complement of the union of $D_{i j} \cap D_{k l}$ for all the irreducible divisors of $D$. Since the latter set is of codimension two, the right-hand side of (10.4) is holomorphic everywhere [18]. By Theorem 9.7 the two sides of (10.3) are proportional to each other. The proportionality constant is easily found to be unity by putting e.g. $Q_{2}=P_{2}$.

Remark 10.3. Geometers usually prefer to multiply out the denominators in (10.4). The trisecant identity then takes the form:

$$
\begin{aligned}
& \theta[\alpha]\left(Q_{1}-P_{1}\right) \theta[\alpha]\left(Q_{2}-P_{2}\right) E\left(Q_{1}, P_{2}\right) E\left(P_{1}, Q_{2}\right) \\
& \quad+\theta[\alpha]\left(Q_{1}-P_{2}\right) \theta[\alpha]\left(Q_{2}-P_{1}\right) E\left(Q_{1}, P_{1}\right) E\left(Q_{2}, P_{2}\right) \\
& \quad=\theta[\alpha]\left(Q_{1}+Q_{2}-P_{1}-P_{2}\right) \theta[\alpha](0) E\left(Q_{1}, Q_{2}\right) E\left(P_{1}, P_{2}\right) .
\end{aligned}
$$

\section{The $2 n$-Point Function and the General Fay Identity}

The analysis of the $2 n$-point function $\left\langle\tilde{\psi}\left(Q_{1}\right) \psi\left(P_{1}\right) \ldots \tilde{\psi}\left(Q_{n}\right) \psi\left(P_{n}\right)\right\rangle$ proceeds exactly as for the four-point case. Since no new methods are required, we merely state the main steps.

From the local operator product expansion (2.3), we find that the $2 n$-point function vanishes when $Q_{i}=Q_{j}$ or $P_{i}=P_{j}$ for some $i \neq j \in\{1, \ldots, n\}$ and has a pole when $Q_{i}=P_{j}$ for some $i, j \in\{1, \ldots, n\}$. Taking $2 n$ copies $M_{1}, \ldots, M_{2 n}$ of $M$, these zeros and poles define divisors $D_{z}^{2 n}, D_{p}^{2 n}$ in $M^{2 n} \equiv M_{1} \times \ldots \times M_{2 n}$. We define the total divisor as

$$
D^{2 n}=D_{p}^{2 n}-D_{z}^{2 n} \text {. }
$$

Let $\psi_{\xi}$ denote the map $M^{2 n} \rightarrow \operatorname{Pic}^{g-1}(M)$ defined by

$$
\left(Q_{1}, P_{1}, \ldots, Q_{n}, P_{n}\right) \rightarrow \mathcal{O}\left(Q_{1}-P_{1}+\ldots+Q_{n}-P_{n}\right) \otimes \xi,
$$

where $\xi \in \operatorname{Pic}^{g-1}(M)$. Let $L_{\xi}^{2 n}$ denote the pullback onto $M^{2 n}$ of $\mathcal{O}(\mathscr{E})$ by this map. Let $F_{\xi}^{2 n}$ denote the line bundle

$$
p_{1}^{*}\left(K \otimes \xi^{-1}\right) \otimes p_{2}^{*}(\xi) \otimes \ldots \otimes p_{2 n-1}^{*}\left(K \otimes \xi^{-1}\right) \otimes p_{2 n}^{*}(\xi),
$$


where $p_{i}: M^{2 n} \rightarrow M$ is the canonical map

$$
\left(z_{1}, \ldots, z_{i}, \ldots, z_{2 n}\right) \rightarrow z_{i} \quad(i=1, \ldots, 2 n) .
$$

Theorem 11.1.

$$
L_{\xi}^{2 n}=F_{\xi}^{2 n} \otimes \mathcal{O}\left(D^{2 n}\right)
$$

Proof. We use the seesaw principle (Remark 4.3) with $X=M_{1} \times \ldots \times M_{2 n-2}$, $Y=M_{2 n-1} \times M_{2 n}$. We carry out the reduction of (11.1) to $X \times\left\{\left(Q_{n}, P_{n}\right)\right\}$ since the argument involves induction. (The other side can be done as before.)

$$
\begin{gathered}
F_{\xi}^{2 n} \mid X \times\left\{\left(Q_{n}, P_{n}\right)\right\}=F_{\xi}^{2 n-2}, \\
\mathcal{O}\left(D^{2 n}\right) \mid X \times\left\{\left(Q_{n}, P_{n}\right)\right\}= \\
\mathcal{O}\left(D^{2 n-2}\right) \otimes p_{1}^{*}\left(J^{-1}\right) \otimes p_{2}^{*}(J) \\
\otimes \ldots \otimes p_{2 n-2}(J),
\end{gathered}
$$

where the $p_{i}$ are now projections from $X=M^{2 n-2}$, and $J=\mathcal{O}\left(Q_{n}-P_{n}\right)$. Thus the right-hand side restricts to

$$
F_{\xi \otimes J}^{2 n-2} \otimes \mathcal{O}\left(D^{2 n-2}\right) \text {. }
$$

It is easily seen that $L_{\xi}^{2 n}$ restricts to $L_{\xi \otimes J}^{2 n-2}$. Hence if Theorem 11.1 holds for $n-1$, it holds for $n$ on restriction to $X \times\left\{\left(Q_{n}, P_{n}\right)\right\}$. For the other side of the seesaw induction is not required.

The proof of the uniqueness of the holomorphic section of $L_{\xi}^{2 n}$ with $\xi=\alpha$ proceeds exactly as in Sect.9. Proposition 9.2 holds and the commutative diagram of Proposition 9.5 holds with obvious changes. We thus get $h^{0}\left(D_{p}^{2 n}, L_{\alpha}^{2 n} \mid D_{p}^{2 n}\right)=1$, which implies:

Theorem 11.2. If $\alpha \in \mathrm{Pic}^{g-1}(M)$ is such that $H^{0}(M, \alpha)=0$, then

$$
h^{0}\left(M^{2 n}, L_{\alpha}^{2 n}\right)=1 .
$$

Writing down the unique holomorphic section of $L_{\alpha}^{2 n}$ and the unique meromorphic of $\mathcal{O}\left(-D^{2 n}\right)$ with divisor $-D^{2 n}$ we obtain:

Theorem 11.3. If $\alpha \in \operatorname{Pic}^{g-1}(M)$ is such that $H^{0}(M, \alpha)=0$ so that $\theta[\alpha](0) \neq 0$, then the unique normalised $2 n$-point correlation function $\left\langle\tilde{\psi}\left(Q_{1}\right) \psi\left(P_{1}\right) \ldots \tilde{\psi}\left(Q_{n}\right) \psi\left(P_{n}\right)\right\rangle$ is given by

$$
\frac{\theta[\alpha]\left(\sum_{1}^{n} Q_{i}-\sum_{1}^{n} P_{i}\right)}{\theta[\alpha](0)} \frac{\prod_{i<j} E\left(Q_{i}, Q_{j}\right) E\left(P_{j}, P_{i}\right)}{\prod_{i, j} E\left(Q_{i}, P_{j}\right)} .
$$

We can also write down the correlation function as a determinant of two-point functions ("Wick's theorem") and verify that it is indeed a meromorphic section of $F_{\alpha}^{2 n}$ with the correct local singularity structure. Then the same argument used to prove Theorem 10.2 gives us:

Theorem 11.4 (Fay's general identity). Let $\alpha \in \operatorname{Pic}^{g-1}(M)$ be such that $H^{0}(M, \alpha)=0$ so that $\theta[\alpha](0) \neq 0$. Then,

$$
\frac{\theta[\alpha]\left(\sum_{1}^{n} Q_{i}-\sum_{1}^{n} P_{j}\right)}{\theta[\alpha](0)} \frac{\prod_{i<j} E\left(Q_{i}, Q_{j}\right) E\left(P_{j}, P_{i}\right)}{\prod_{i, j} E\left(Q_{i}, P_{j}\right)}=\operatorname{det}\left[S_{\alpha}\left(Q_{i}, P_{j}\right)\right] .
$$


Remark 11.5. The main result of this section is that we are able to show by a direct proof that the $2 n$-point function is unique. The general Fay identity of Theorem 11.4 is itself, as remarked by Fay [1], a consequence of the trisecant identity (Theorem 10.2).

Acknowledgement. I thank Prof. M. S. Narasimhan for many useful discussions.

\section{References}

1. Fay, J.D.: Theta functions on Riemann surfaces. Berlin, Heidelberg, New York: Springer 1973

2. Mumford, D.: Tata lectures on Theta. II. Boston, Basel, Stuttgart: Birkhäuser 1984

3. Arbarello, E.: Fay's trisecant formula and a characterization of Jacobian varieties, Rome preprint 1987

Beauville, A.: Le problème de Schottky et la conjecture de Novikov. Séminaire Bourbaki no. 675: Astérisque 152-153, 101-112 (1987); and references therein

4. Eguchi, T., Ooguri, H.: Chiral bosonization on a Riemann surface. Phys. Lett. B 187, 127-134 (1987)

Verlinde, E., Verlinde, H.: Chiral bosonization, determinants and the string partition function. Nucl. Phys. B 288, 357-396 (1987)

5. Witten, E.: Quantum field theory. Grassmannians, and algebraic curves. Commun. Math. Phys. 113, 529-600 (1988)

6. Sen, S., Raina, A.K.: Grassmannians, multiplicative Ward identities and theta function identities. Phys. Lett. B 203, 256-262 (1988)

7. Raina, A.K.: The divisor group and multiplicative Ward identities in conformal field theory. In: Superstring theory. Ramachandran, R., Mani, H.S. (eds.). Singapore: World Scientific 1988

8. Friedan, D., Martinec, E., Shenker, S.: Conformal invariance, supersymmetry, and string theory. Nucl. Phys. B 271, 93-165 (1986)

9. Griffiths, P., Harris, J.: Principles of algebraic geometry. New York: Wiley 1978

10. Hartshorne, R.: Algebraic geometry. Berlin, Heidelberg, New York: Springer 1977

11. Atiyah, M.F.: Riemann surfaces and spin structures. Ann. Sci. Ecole Norm. Sup. 4, 47-62 (1971)

12. Narasimhan, M.S., Ramanan, S.: Moduli of vector bundles on a compact Riemann surface. Ann. Math. 89, 14-51 (1969)

13. Mumford, D.: Abelian varieties. Second Edition. Bombay, London: T.I.F.R. and Oxford University Press 1974

14. Shafarevich, I.R.: Basic algebraic geometry. Berlin, Heidelberg, New York: Springer 1977

15. Bredon, G.E.: Sheaf theory. New York: McGraw-Hill 1967

16. Clemens, C.H.: A Scrapbook of complex curve theory. New York, London: Plenum Press 1980

17. Namazie, M.A., Narain, K.S. Sarmadi, M.H.: Fermionic string loop amplitudes with external bosons. Phys. Lett. B 177, 329-334 (1986);

Sonoda, H.: Calculation of a propagator on a Riemann surface. Phys. Lett. B 178, 390-394 (1986)

18. Bochner, S., Martin, W.T.: Several complex variables. Princeton, NJ: Princeton University Press 1948

Communicated by J. Fröhlich

Received July 5, 1988

Note added in proofs. By the methods of this paper we can easily obtain the remaining correlation functions of the system. Full details appear in a contribution to the Proceedings of the Indo-French Conference on Geometry, Bombay (1989), preprint available on request. 
